\title{
The Usefulness of Clinical-Practice-Based Laboratory Data in Facilitating the Diagnosis of Dengue Illness
}

\author{
Jien-Wei Liu,, ${ }^{1,2,3}$ Ing-Kit Lee, ${ }^{1,2,3}$ Lin Wang, ${ }^{4}$ Rong-Fu Chen, ${ }^{5}$ and Kuender D. Yang ${ }^{5}$ \\ ${ }^{1}$ Division of Infectious Diseases, Department of Internal Medicine, Kaohsiung Chang Gung Memorial Hospital, Kaohsiung 833, Taiwan \\ ${ }^{2}$ Infection Control Team, Kaohsiung Chang Gung Memorial Hospital, Kaohsiung 833, Taiwan \\ ${ }^{3}$ Chang Gung University College of Medicine, Tao-Yuan 333, Taiwan \\ ${ }^{4}$ Department of Pediatrics, Kaohsiung Chang Gung Memorial Hospital, Kaohsiung 833, Taiwan \\ ${ }^{5}$ Department of Medical Research, Show Chwan Memorial Hospital-Chang Bing, Changhua 500, Taiwan
}

Correspondence should be addressed to Jien-Wei Liu; jwliu@cgmh.org.tw

Received 13 May 2013; Accepted 23 October 2013

Academic Editor: Vittorio Sambri

Copyright ( 2013 Jien-Wei Liu et al. This is an open access article distributed under the Creative Commons Attribution License, which permits unrestricted use, distribution, and reproduction in any medium, provided the original work is properly cited.

\begin{abstract}
Alertness to dengue and making a timely diagnosis is extremely important in the treatment of dengue and containment of dengue epidemics. We evaluated the complementary role of clinical-practice-based laboratory data in facilitating suspicion/diagnosis of dengue. One hundred overall dengue (57 dengue fever [DF] and 43 dengue hemorrhagic fever [DHF]) cases and another 100 nondengue cases (78 viral infections other than dengue, 6 bacterial sepsis, and 16 miscellaneous diseases) were analyzed. We separately compared individual laboratory variables (platelet count $[\mathrm{PC}]$, prothrombin time $[\mathrm{PT}]$, activated partial thromboplastin time [APTT], alanine aminotransferase [ALT], and aspartate aminotransferase [AST]) and varied combined variables of DF and/or DHF cases with the corresponding ones of nondengue cases. The sensitivity, specificity, accuracy, positive predictive value (PPV), and negative predictive value (NPV) in the diagnosis of DF and/or DHF were measured based on these laboratory variables. While trade-off between sensitivity and specificity, and/or suboptimal PPV/NPV was found at measurements using these variables, prolonged APTT + normal PT $+\mathrm{PC}<100 \times 10^{9}$ cells/L had a favorable sensitivity, specificity, PPV, and NPV in diagnosis of DF and/or DHF. In conclusion, these data suggested that prolonged APTT + normal PT $+\mathrm{PC}<100 \times 10^{9}$ cells/L is useful in evaluating the likelihood of DF and/or DHF.
\end{abstract}

\section{Introduction}

Dengue is a major medical and public health problem in tropical and subtropical regions. It is estimated that more than 2.5 billion people are living in geographic locales where dengue is endemic, and 50-100 million people have been annually infected by dengue virus (DENV) [1]. The spectrum of clinical manifestations of dengue ranges from a mild-form nonspecific febrile illness, classic dengue fever (DF), to the severe-form dengue hemorrhagic fever (DHF) $[2,3]$. DHF is characterized by the presence of hemorrhagia, thrombocytopenia $\left(<100 \times 10^{9}\right.$ cells/L), and clinical evidence of plasma leak resulting from increased vascular permeability $[2,3]$. Based on the World Health Organization (WHO) criteria, the severity of DHF was categorized into grades I-IV as follows.
DHF grade I is manifested by fever accompanied by nonspecific constitutional symptoms, with a positive tourniquet test result; DHF grade II is the appearance of spontaneous bleeding in addition to constitutional symptoms; DHF grade III is circulatory failure with signs of rapid and weak pulse, narrowing of pulse pressure or hypotension, and the presence of cold clammy skin; and DHF grade IV is profound shock with undetectable blood pressure and pulse [4]. Grades III and IV are grouped as dengue shock syndrome (DSS) [4]. The definitive diagnosis of dengue illness is made by positive result(s) of serology testing [5], and these serology tests are unfortunately not always readily available at most clinical laboratories. As a result, the clinically mild-form dengue has been inevitably underreported $[6,7]$, and it is uncommon that clinicians fail to make a timely detection of the early stage of 
a dengue before it evolves into an overt clinical severe-form DHF. Clinicians inexperienced with dengue may not be alert to this infection entity, and this is especially true for clinicians in a nondengue endemic setting. Once DSS developed, the mortality rate in the affected patients might soar to as high as $20 \%$ [8]. It is not uncommon that dengue outbreaks are recognized only when hundreds of people are affected [7], making containment of dengue epidemics difficult and challenging. The importance of a timely diagnosis of dengue illness cannot be overemphasized.

Dengue epidemic was once absent in Taiwan after 1942 $[9,10]$. It was not until the 1980s that a number of dengue epidemics reemerged, and of them, two remarkably large ones occurred in 1988 and 2002 in the southern part of this island $[9,10]$. The rest were sporadic dengue clusters, and there was a small number of silent dengue transmissions between some of these dengue clusters $[11,12]$. Owing to the absence of large-scale dengue epidemics like those annually found in southeastern Asian countries [2], most clinicians in Taiwan are not experienced with dengue illness.

In 2002 a dengue epidemic due to dengue virus serotype 2 (DENV-2) developed in southern Taiwan in which more than 5000 symptomatic cases were found and most of the affected patients were adults $[10,13]$, and thanks to the convenience of medical access in Taiwan, a large number of febrile patients presented to Emergency Services of Kaohsiung Chang Gung Memorial Hospital (KSCGMH) seeking medical help because of their concern for possible dengue illness. KSCGMH is a 2500-bed medical facility serving as a primary care and tertiary referral centre in this area. Complying with the law, clinicians notified Center for Disease Control (CDC, Taiwan) of patients with suspected DF/DHF and sampled patients' blood specimens for the central laboratory of CDC for serologic confirmation of dengue. Of note, blood specimens of a substantial number of suspicious dengue patients were also subject to clinicalpractice-based laboratory investigations/tests such as bacterial culture, complete blood count (CBC), coagulation tests, and blood chemistry analysis, as the diagnoses of dengue were doubtful and further testing to exclude other diseases was therefore needed. To elucidate whether the clinicalpractice-based laboratory data play a complementary role in facilitating the diagnosis of DF/DHF, the aims of this study were to estimate the sensitivity, specificity, accuracy, positive predictive value (PPV), and negative predictive value (NPV) of the individual data and varied combinations of each of these data retrieved from hemogram (i.e., peripheral white cell count [WBC], platelet count $[\mathrm{PC}])$, coagulation profile (i.e., prothrombin time [PT], activated partial thromboplastin time [APTT]), and blood chemistry (i.e., alanine aminotransferase [ALT] and aspartate aminotransferase [AST]) in the diagnosis of DF/DHF. The above-mentioned data were selected for analyses, because with the exception of PT, they were frequently found to be abnormal in dengue-affected patients [10,13-17]. The results of this study provide valuable information that potentially helps build up a justified suspicious index for $\mathrm{DF} / \mathrm{DHF}$ and/or facilitates the diagnosis of $\mathrm{DF} / \mathrm{DHF}$ before the results of serological tests for dengue are available.

\section{Patients and Methods}

Potentially eligible included patients who were adults aged $\geqq 18$ years with a tentative clinical diagnosis of $\mathrm{DF} / \mathrm{DHF}$ treated at Emergency Services or during admission at KSCGMH between July and November 2002. After excluding those with missing data, a total of 200 patients were eventually included for analyses. Half of the included patients who were subsequently proven to be serologically dengue-positive were allocated as the overall dengue cases, while another half who were serologically dengue-negative were allocated as the nondengue cases. A definitive diagnosis of dengue was made when at least one of the following serologic test results was found: (i) a positive reverse-transcriptase polymerase detection of DENV, (ii) a positive enzyme-linked immunosorbent assay result for specific immunoglobulin $\mathrm{M}$ antibody for DENV in acute-phase serum, and (iii) a fourfold or higher increase in dengue-specific hemagglutination inhibition titers in convalescent serum as compared to that in acute-phase serum [13-16]. The serologic tests for DENV infection were performed by CDC, Taiwan. Patients of the overall dengue cases were separated into those with DF (DF cases) and those with DHF (DHF cases) for further analyses [4].

A retrospective chart review was carried out for collection of demographic, clinical, and clinical-practice-based laboratory information of the included patients. The retrieved WBC, PC, PT, APTT, ALT, and AST were assayed from the specimens sampled from the affected patients when the tentative diagnosis of DF/DHF was made. Each of these data was regarded as an individual variable. Each individual variable and varied combined variables of the overall dengue cases, DF cases, and DHF cases were separately compared to the corresponding ones of nondengue cases. Individual components in combined variables were results of assays of blood specimens sampled on the same day. Because a large number of variables were derived from combination of individual variables and because each of these combined variables were regarded as one individual variable in analysis, to make the complexity more legible, combined variable $\mathrm{A}$ and variable $B$ were expressed as a variable $A+B$ and so on. The Chi-squared test or Fisher exact test was used for comparison of dichotomous variables, while the Student's $t$ test or Mann-Whitney $U$ test was used for comparison of continuous variables. A 2 -tailed $P$ was considered statistically significant.

To assess the values of the aforementioned data in facilitating the suspicion/diagnoses of dengue in general and DF/DHF in particular, we calculated the sensitivity, specificity, accuracy, PPV, and NPV based on these individual laboratory variables and varied combinations of each of them [18]. Because of the trade-off between the measurements of sensitivity and specificity, and because of variables with high accuracy suggesting the coexistence of a potentially acceptable sensitivity and specificity, individual laboratory variables and combined variables with a calculated accuracy $>0.80$ in the diagnosis and DF and/or DHF would be further examined with the receiver-operating-characteristic (ROC) curve analysis plotting sensitivity against 1-specificity 
TABLE 1: Demographic and clinical information of the included patients.

\begin{tabular}{|c|c|c|c|c|c|c|c|}
\hline Variable & $\begin{array}{c}\text { Overall dengue } \\
\text { cases (A) } \\
(N=100)\end{array}$ & $P($ A versus $\mathrm{D})$ & $\begin{array}{l}\text { Dengue fever } \\
\text { cases (B) } \\
(N=57)\end{array}$ & $P(\mathrm{~B}$ versus $\mathrm{D})$ & $\begin{array}{c}\text { Dengue } \\
\text { hemorrhagic } \\
\text { cases }(\mathrm{C}) \\
(N=43)\end{array}$ & $P(\mathrm{C}$ versus $\mathrm{D})$ & $\begin{array}{l}\text { Nondengue } \\
\text { cases }^{1}(\mathrm{D}) \\
(N=100)\end{array}$ \\
\hline \multicolumn{8}{|l|}{ Demographics } \\
\hline Age, yr & & 0.375 & & 0.669 & & 0.279 & \\
\hline Mean $( \pm \mathrm{SD})$ & $46.1 \pm 11.5$ & & $45.1 \pm 12.3$ & & $41.2 \pm 10.5$ & & $44.9 \pm 17.6$ \\
\hline Median (range) & $49(18-68)$ & & $47(18-68)$ & & $49(18-63)$ & & $43(18-81)$ \\
\hline Male gender, no. (\%) & $44(44)$ & 0.667 & $22(38.6)$ & $>0.99$ & $22(51.2)$ & 0.270 & $40(40)$ \\
\hline \multicolumn{8}{|l|}{$\begin{array}{l}\text { Underlying } \\
\text { condition, }{ }^{2} \text { no. (\%) }\end{array}$} \\
\hline Hypertension & $13(13)$ & $>0.99$ & $6(10.5)$ & 0.801 & $7(16.3)$ & 0.607 & $13(13)$ \\
\hline Diabetes mellitus & $14(14)$ & 0.834 & $5(8.8)$ & 0.603 & $9(20.9)$ & 0.199 & $12(12)$ \\
\hline Old stroke & 0 & 0.121 & 0 & 0.297 & 0 & 0.316 & $4(4)$ \\
\hline $\begin{array}{l}\text { Chronic kidney } \\
\text { disease }\end{array}$ & 0 & 0.121 & 0 & 0.297 & 0 & 0.316 & $4(4)$ \\
\hline Solid tumor & 0 & 0.246 & 0 & 0.554 & 0 & 0.554 & $3(3)$ \\
\hline \multicolumn{8}{|l|}{$\begin{array}{l}\text { Symptom/sign, } \\
\text { no. (\%) }\end{array}$} \\
\hline Fever & $96(96)$ & 0.251 & $57(100)$ & 0.027 & $39(90.7)$ & $>0.99$ & $91(91)$ \\
\hline Bone pain & $55(55)$ & 0.007 & $33(57.9)$ & 0.007 & $22(51.2)$ & 0.093 & $35(35)$ \\
\hline Retroorbital pain & $8(8)$ & $>0.99$ & $6(10.5)$ & 0.782 & $2(4.7)$ & 0.505 & $9(9)$ \\
\hline Arthralgia & $10(10)$ & 0.033 & $6(10.5)$ & 0.027 & $4(9.3)$ & 0.067 & $2(2)$ \\
\hline Abdominal pain & $40(40)$ & 0.1 & $20(35.1)$ & 0.373 & $20(46.5)$ & 0.036 & $28(28)$ \\
\hline Cough & $31(31)$ & 0.342 & $16(28.1)$ & 0.574 & $15(34.9)$ & 0.220 & $24(24)$ \\
\hline Diarrhea & $22(22)$ & 0.197 & $11(19.3)$ & 0.497 & $11(25.6)$ & 0.148 & $14(14)$ \\
\hline Nausea/vomiting & $36(36)$ & 0.042 & $19(33.3)$ & 0.134 & $17(39.5)$ & 0.041 & $22(22)$ \\
\hline Rash & $34(34)$ & 0.005 & $24(42.1)$ & 0.001 & $10(23.3)$ & 0.347 & $16(16)$ \\
\hline Myalgia & $15(15)$ & $<0.001$ & $10(17.5)$ & $<0.001$ & $5(11.6)$ & $<0.001$ & $47(47)$ \\
\hline Petechiae & $44(44)$ & $<0.001$ & $19(33.3)$ & $<0.001$ & $25(58.1)$ & $<0.001$ & $9(9)$ \\
\hline Gum bleeding & $26(26)$ & $<0.001$ & $13(22.8)$ & $<0.001$ & $13(30.2)$ & $<0.001$ & 0 \\
\hline $\begin{array}{l}\text { Gastrointestinal } \\
\text { bleeding }\end{array}$ & $20(20)$ & $<0.001$ & $7(12.3)$ & 0.001 & $13(30.2)$ & $<0.001$ & 0 \\
\hline
\end{tabular}

${ }^{1}$ Including viral infections other than dengue illness (78 cases), bacterial sepsis ( 6 cases), gastrointestinal bleeding with/without liver cirrhosis (4 cases), urinary tract infection ( 2 cases), and aplastic anemia, colitis, acute hepatitis, acute pancreatitis, hepatocellular carcinoma, biliary tract infection, aseptic meningitis, systemic lupus erythematosus, acute tonsillitis, and pneumonia (each 1 case).

${ }^{2}$ An individual patient might have more than one underlying condition.

${ }^{3}$ An individual patient might have more than one symptom/sign.

$[19,20]$. The area under the curve (AUC) with its 95\% confidence interval of each separately constructed ROC curve was measured using SPSS 15 software for Windows (SPSS Inc., Chicago, Ill) to obtain the predictive accuracy of these clinical-practice-based laboratory data in the diagnosis of DF and/or DHF. AUC between 0.90 and 1 was considered excellent, between 0.80 and 0.90 good, between 0.70 and 0.80 fair, between 0.60 and 0.70 poor, and between 0.50 and 0.60 fail [20].

\section{Results}

3.1. Demographic, Clinical, and Laboratory Information of Patients in Dengue and Nondengue Cases. The overall dengue case and nondengue case groups each included 100 patients, and the former was made up of 57 DF cases and 43 DHF cases. Similar demographics but a number of significant differences in clinical manifestations (Table 1) and clinicalpractice-based laboratory data (Table 2) were found when the overall dengue cases, DF cases, and DHF cases were separately compared with the nondengue cases.

3.2. Sensitivity, Specificity, PPV, NPV, and Accuracy of Laboratory Data in the Diagnoses of the Overall Dengue, DF, and DHF. The sensitivity, specificity, PPV, NPV and accuracy calculated from individual laboratory variables and varied combinations of them in the diagnosis of DF and/or DHF are summarized in Table 3. 
TABLE 2: Laboratory data of the included patients.

\begin{tabular}{|c|c|c|c|c|c|c|c|}
\hline Variable & $\begin{array}{l}\text { Overall dengue } \\
\text { cases (A) } \\
(N=100)\end{array}$ & $P(\mathrm{~A}$ versus $\mathrm{D})$ & $\begin{array}{c}\text { Dengue fever } \\
\text { cases }(\mathrm{B}) \\
(N=57)\end{array}$ & $P(\mathrm{~B}$ versus $\mathrm{D})$ & $\begin{array}{c}\text { Dengue } \\
\text { hemorrhagic } \\
\text { cases }(\mathrm{C}) \\
(N=43)\end{array}$ & $P(\mathrm{C}$ versus $\mathrm{D})$ & $\begin{array}{l}\text { Nondengue } \\
\text { cases }^{1}(\mathrm{D}) \\
(N=100)\end{array}$ \\
\hline $\begin{array}{l}\text { Leukopenia }(\mathrm{WBC} \\
<3.0 \times 10^{9} \text { cells/L), no. } \\
(\%)\end{array}$ & $49(40)$ & 0.001 & $31(54.4)$ & $<0.001$ & $18(41.9)$ & 0.049 & $25(25)$ \\
\hline $\begin{array}{l}\text { Platelet count }<150.0 \\
\left(\times 10^{9} \text { cells } / \mathrm{L}\right), \text { no. }(\%)\end{array}$ & $100(100)$ & $<0.001$ & $57(100)$ & $<0.001$ & $43(100)$ & $<0.001$ & $71(71)$ \\
\hline $\begin{array}{l}\text { Platelet count }<100.0 \\
\left(\times 10^{9} \text { cells } / \mathrm{L}\right), \text { no. }(\%)\end{array}$ & $97(97)$ & $<0.001$ & $54(94.7)$ & $<0.001$ & $43(100)$ & $<0.001$ & $30(30)$ \\
\hline $\begin{array}{l}\text { Prolonged APTT, } \\
n / N(\%)\end{array}$ & 61/68 (89.7) & 0.001 & $33 / 36$ (91.7) & 0.006 & 28/32 (87.5) & 0.030 & $26 / 41(63.4)$ \\
\hline $\begin{array}{l}\text { Prolonged } \mathrm{PT}^{3}, n / N \\
(\%)\end{array}$ & $1 / 68(1.5)$ & 0.023 & $0 / 37$ & 0.055 & $1 / 31(3.2)$ & 0.217 & $5 / 39(12.8)$ \\
\hline $\begin{array}{l}\mathrm{AST}>40 \mathrm{U} / \mathrm{L} \text { (normal } \\
\text { value, }<40 \mathrm{U} / \mathrm{L}), n / N \\
(\%)\end{array}$ & $68 / 79$ (86.1) & $<0.001$ & $34 / 44$ (77.3) & $<0.001$ & $34 / 35$ (97.1) & $<0.001$ & $27 / 64(42.2)$ \\
\hline $\begin{array}{l}\mathrm{ALT}>40 \mathrm{U} / \mathrm{L} \text { (normal } \\
\text { value, }<40 \mathrm{U} / \mathrm{L}), n / N \\
(\%)\end{array}$ & $49 / 65(75.4)$ & $<0.001$ & $24 / 37$ (64.9) & 0.006 & $25 / 28(89.3)$ & $<0.001$ & $19 / 55(34.5)$ \\
\hline
\end{tabular}

Abbreviations: APTT: activated partial thromboplastin time; PT: prothrombin time; AST: aspartate aminotransferase; ALT: alanine aminotransferase; $n / N$ : no. of patients/no. of patients with data available.

${ }^{1}$ See footnote in Table 1 for details.

${ }^{2}$ Prolonged APTT was defined as an increased APTT value $>20 \%$ of the control value.

${ }^{3}$ Prolonged PT was defined as an increased PT value $>3$ seconds than that of control.

In the diagnosis of overall dengue, variables with a high sensitivity, in decreasing order, were $\mathrm{PC}<150 \times 10^{9}$ cells/L (100\%), PC $<100 \times 10^{9}$ cells/L (97\%), prolonged APTT (89.7\%), prolonged APTT $+\mathrm{PC}<150 \times 10^{9}$ cells/L $(89.6 \%)$, prolonged APTT + normal PT (89.4\%), prolonged APTT + normal PT $+\mathrm{PC}<150 \times 10^{9}$ cells/L $(89.4 \%)$, prolonged $\mathrm{APTT}+\mathrm{PC}<100 \times 10^{9}$ cells/L $(88.6 \%)$, and prolonged $\mathrm{APTT}+$ normal PT $+\mathrm{PC}<100 \times 10^{9}$ cells/L $(87.9 \%)$. The specificity of $\mathrm{PC}<150 \times 10^{9}$ cells/L and $\mathrm{PC}<100 \times 10^{9}$ cells/L was $48.8 \%$ and $70 \%$, respectively. Prolonged APTT + normal PT $+\mathrm{PC}<100 \times 10^{9}$ cells/L had an accuracy of $84.6 \%$ (sensitivity $87.9 \%$, specificity $78.9 \%$, PPV $87.9 \%$, and NPV $78.9 \%$ ), while PC $<100 \times 10^{9}$ cells/L had an accuracy of $83.5 \%$ (sensitivity $97.0 \%$, specificity $70.0 \%$, PPV $76.4 \%$, and NPV 95.9\%).

In the diagnosis of $\mathrm{DF}$, variables with a high sensitivity, in decreasing order, were $\mathrm{PC}<150 \times 10^{9}$ cells/L $(100 \%)$, PC $<$ $100 \times 10^{9}$ cells $/ \mathrm{L}(94.7 \%)$, prolonged APTT + normal PT + $\mathrm{PC}<150 \times 10^{9}$ cells/L $(91.7 \%)$, prolonged APTT $(91.7 \%)$, prolonged APTT + normal PT (91.7\%), prolonged APTT + $\mathrm{PC}<100 \times 10^{9}$ cells/L (88.9\%), and prolonged APTT + normal PT $+\mathrm{PC}<100 \times 10^{9}$ cells/L $(88.9 \%)$. Among them, prolonged APTT + normal PT $+\mathrm{PC}<100 \times 10^{9}$ cells $/ \mathrm{L}$ had an accuracy of $83.8 \%$ (specificity $78.9 \%$, PPV $80 \%$, and NPV $88.2 \%$ ). The specificity of $\mathrm{PC}<150 \times 10^{9}$ cells/L and PC $<$ $100 \times 10^{9}$ cells/L was $29.0 \%$ and $70 \%$, respectively.

In the diagnosis of DHF, variables with high sensitivity, in decreasing order, were $\mathrm{PC}<150 \times 10^{9}$ cells/L $(100 \%)$, $\mathrm{PC}<100 \times 10^{9}$ cells/L (100\%), AST $>40 \mathrm{U} / \mathrm{L}$ (97.1\%), PC
$<150 \times 10^{9}$ cells $/ \mathrm{L}+\mathrm{AST}>40 \mathrm{U} / \mathrm{L}(97.1 \%), \mathrm{PC}<100 \times$ $10^{9}$ cells $/ \mathrm{L}+\mathrm{AST}>40 \mathrm{U} / \mathrm{L}(97.1 \%), \mathrm{ALT}>40 \mathrm{U} / \mathrm{L}+\mathrm{AST}>$ $40 \mathrm{U} / \mathrm{L}$ (92.6\%), PC $<150 \times 10^{9}$ cells/L + ALT $>40 \mathrm{U} / \mathrm{L}+$ AST $>40 \mathrm{U} / \mathrm{L}(92.6 \%)$, and $\mathrm{PC}<100 \times 10^{9}$ cells $/ \mathrm{L}+\mathrm{ALT}>$ $40 \mathrm{U} / \mathrm{L}+\mathrm{AST}>40 \mathrm{U} / \mathrm{L}$ (92.6\%). The specificity of PC $<$ $150 \times 10^{9}$ cells/L and $\mathrm{PC}<100 \times 10^{9}$ cells/L was $29 \%$ and $70 \%$, respectively. Variables with high accuracy $(>80.0 \%)$ were $\mathrm{PC}<100 \times 10^{9}$ cells $/ \mathrm{L}+\mathrm{ALT}>40 \mathrm{U} / \mathrm{L}+\mathrm{AST}>$ $40 \mathrm{U} / \mathrm{L}$ (sensitivity $92.6 \%$, specificity $76.6 \%$, PPV $69.4 \%$, and NPV 94.7\%), prolonged APTT + normal PT + PC $<100 \times$ $10^{9}$ cells/L (sensitivity $86.7 \%$, specificity $78.9 \%$, PPV $76.5 \%$, and NPV 88.2\%), prolonged APTT + normal PT + AST > $40 \mathrm{U} / \mathrm{L}$ (sensitivity $88.5 \%$, specificity $75 \%$, PPV $76.7 \%$, and NPV 87.5\%), prolonged APTT + normal PT + ALT > $40 \mathrm{U} / \mathrm{L}$ (sensitivity $81 \%$, specificity $81.5 \%$, PPV $77.3 \%$, and NPV $84.6 \%$ ), and $\mathrm{PC}<100 \times 10^{9}$ cells/L + ALT $>40 \mathrm{U} / \mathrm{L}$ (sensitivity $89.3 \%$, specificity $75.5 \%$, PPV $65.8 \%$, and NPV $93 \%$ ).

3.3. Sensitivity, Specificity, PPV, NPV, Accuracy, and AUC of Laboratory Variables Included in ROC Curve Analysis. PC $<$ $100 \times 10^{9}$ cells $/ \mathrm{L}$, prolonged APTT + normal PT $+\mathrm{PC}<100 \times$ $10^{9}$ cells $/ \mathrm{L}, \mathrm{PC}<100 \times 10^{9}$ cells $/ \mathrm{L}+\mathrm{ALT}>40 \mathrm{U} / \mathrm{L}, \mathrm{PC}<100 \times$ $10^{9}$ cells $/ \mathrm{L}+\mathrm{ALT}>40 \mathrm{U} / \mathrm{L}+\mathrm{AST}>40 \mathrm{U} / \mathrm{L}$, prolonged $\mathrm{APTT}$ + normal PT + AST $>40 \mathrm{U} / \mathrm{L}$, and prolonged APTT + normal $\mathrm{PT}+\mathrm{ALT}>40 \mathrm{U} / \mathrm{L}$ were included for ROC analysis. AUC, along with the sensitivity, specificity, PPV, NPV, and accuracy in the diagnosis of DF and/or DHF is summarized in Table 4. $\mathrm{PC}<100 \times 10^{9}$ cells/L and prolonged APTT + normal PT + $\mathrm{PC}<100 \times 10^{9}$ cells/L each had a good predictive accuracy 







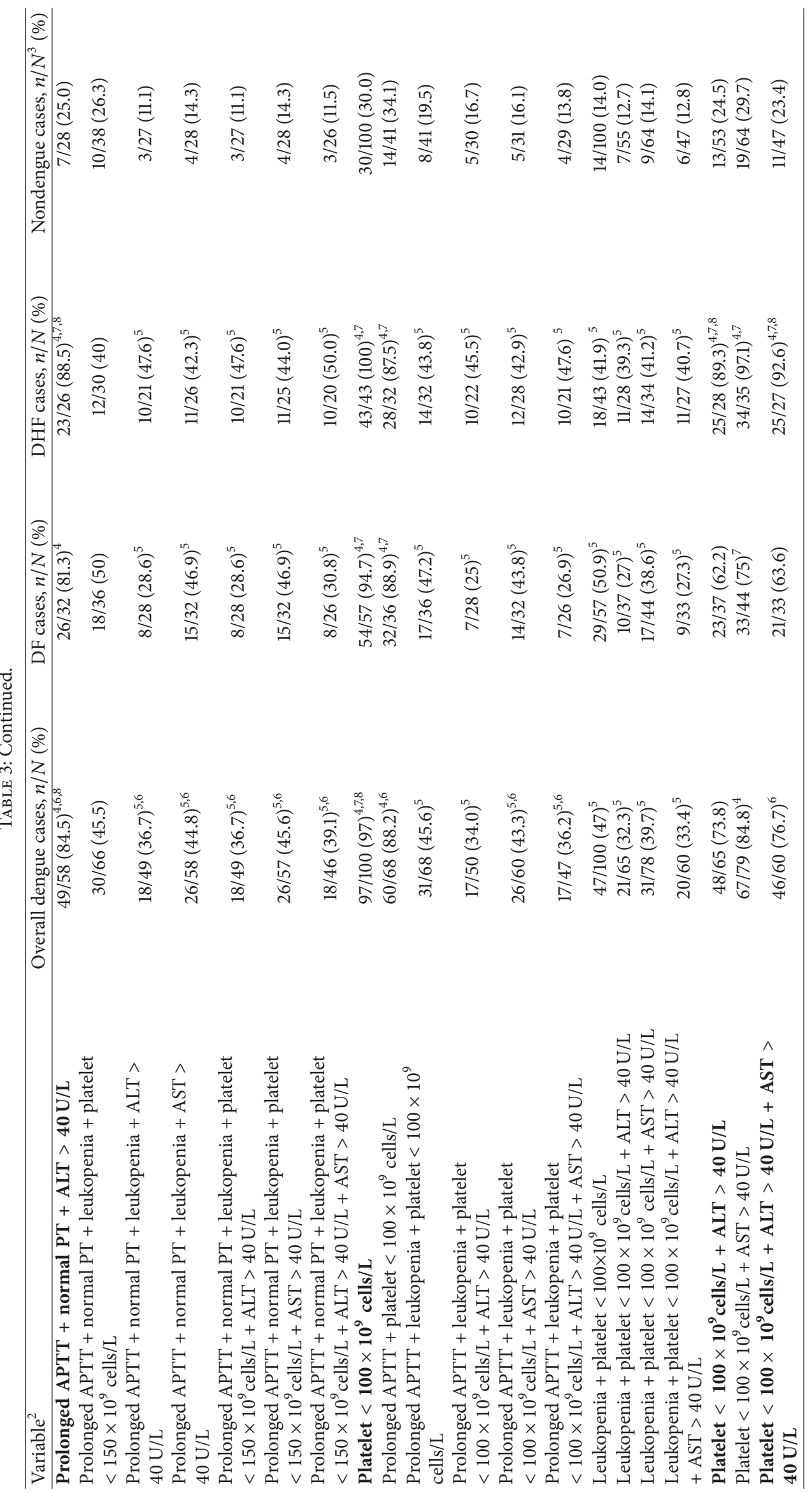




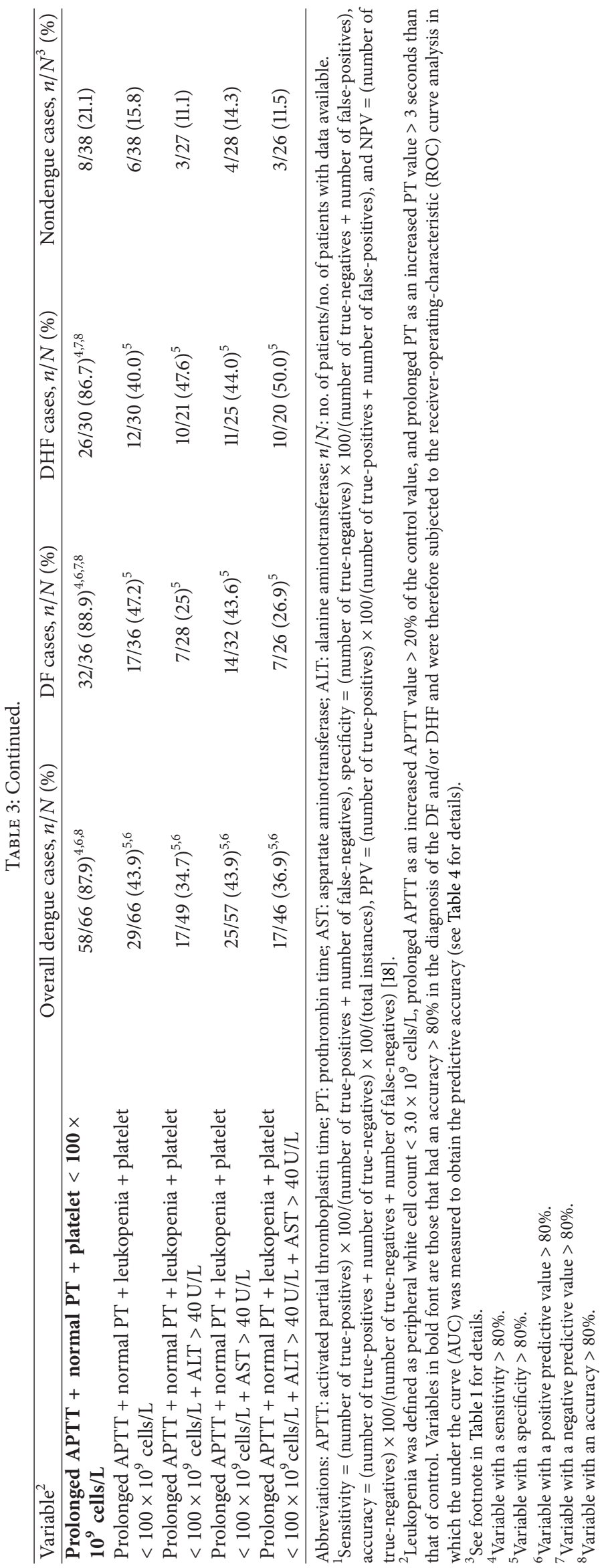




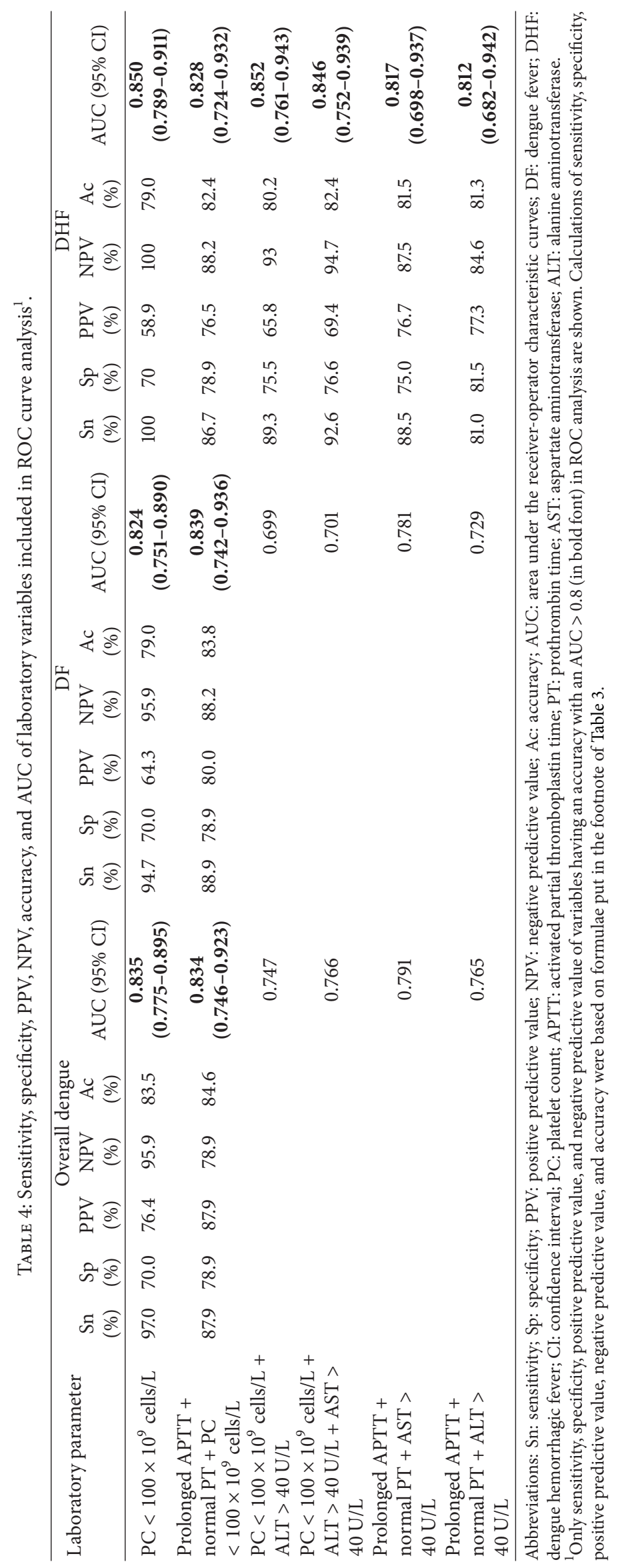


(AUC > 0.8) in the diagnoses of the overall dengue, DF, and DHF, while the remaining variables had a good predictive accuracy only in the diagnosis of DHF.

\section{Discussion}

The immunopathogenesis of DF/DHF is characterized by an aberrant immune overactivation and cytokine overproduction that lead to the development of a great array of clinical and laboratory manifestations [17, 21-23]. Some of the cytokines are proinflammatory, while others are antiinflammatory $[17,21-23]$. These cytokines are capable of causing leukocytes to activate synergistically or antagonistically $[17,24]$, and clinical and laboratory manifestations in the dengue affected patients are the net effect of the interactions between one another among these activated cytokines [17, 2123].

Myelosuppression in DF/DHF leads to leukopenia [24], and some of the dengue-affected patients experience prior transient neutrophilia and monocytosis before development of leukopenia [24]. DEV-2 was reported to be able to bind to human platelets in the presence of virus-specific antibodies [25]. As a result of molecular mimicry, autoantibodies produced in DF/DHF patients are capable of coating human platelets [17], and IFN- $\gamma$ activates macrophages to phagocytosize the auto antibody-coated platelets, rendering thrombocytopenia [24]. During acute DENV infection, both coagulation and fibrinolysis are activated, leading to alterations in coagulation parameters (e.g., platelet count and APTT) and fibrinolytic parameters (e.g., tissue-type plasminogen [tPA] and plasminogen activator inhibitor [tAPI]). APTT prolongs as tPA increases. The activations of coagulation and fibrinolysis are much more drastic in DHF/DSS than in DF [24, 26].

An APTT prolongation and normal PT often found in DHF suggest a defect in the intrinsic pathway of coagulation, which is caused by either downregulation of the synthesis or overconsumption of specific factor(s) that are presumably produced by hepatocytes [24]. Hepatitis is usually found in the acute phase of DF/DHF $[24,27]$. Data derived from the analysis of the linear correlation and regression between the levels of AST/ALT and APTT show a strong association between them, suggesting that hepatic dysfunction might be responsible for the decreased synthesis of specific factors in the coagulation intrinsic pathway $[24,28]$. Increased factor consumption as indicated by the high levels of tPA is also associated with APTT prolongation [26]. Elevated liver enzymes are especially found in patients with DHF [27], and this may explain the findings that variables made up of $\mathrm{PC}<$ $100 \times 10^{9} \mathrm{cells} / \mathrm{L}$ and/or prolonged APTT + normal PT with an elevated AST and/or an elevated ALT had a good accuracy in the diagnosis of DHF but not in the diagnosis of the overall dengue or DF.

Given significant differences in clinical manifestations between the overall dengue/DF/DHF cases and the nondengue cases in this series and in others [29], clinicians experienced with these infectious disease entities may not often have difficulty making the diagnosis of DF/DHF on clinical basis, especially in areas where DF/DHF is always endemic [29]. On the other hand, for inexperienced clinicians the clinical diagnosis of $\mathrm{DF} / \mathrm{DHF}$ is often a big challenge. The scenarios in which clinical-based suspicion/diagnosis of DF/DHF is challenging include inexperienced clinicians' facing febrile patients in a small dengue cluster or encountering febrile travelers from dengue-endemic locales to nondengue-endemic area. The significant differences in the daily-practice-based laboratory data between patients with DF/DHF and the nondengue cases (Table 2) suggested these individual data alone and/or in combination with other(s) potentially facilitate the suspicion/diagnosis of DF/DHF. One study from Singapore where dengue was found all year round reported that a model combining clinical feature (skin rash) and laboratory parameters (white cell count, hemoglobin, PT, creatinine, and bilirubin levels) was able to distinguish dengue illness (mainly DF) from other infections with a sensitivity of $84 \%$ and specificity of $85 \%$ [29].

While ROC plots provide a global comprehensive view of the test, sensitivity and specificity describe the test's ability to correctly distinguish between DF/DHF and nondengue patients [19]. As $\mathrm{PC}<100 \times 10^{9}$ cells/L had a high sensitivity but low specificity in the diagnosis of DF and/or DHF, it may be useful in screening dengue illness when this viral infection is rarely encountered. Prolonged APTT + normal $\mathrm{PT}+\mathrm{PC}<100 \times 10^{9}$ cells/L with high sensitivity $(87.9 \%$ for the overall dengue, $88.9 \%$ for DF, and $86.7 \%$ for DHF) and a comparatively high specificity of $78.9 \%$ for DF and/or DHF in this series suggest that the combined variable is especially useful in screening dengue illness during a dengue epidemic or in countries where dengue is always endemic, as under these circumstances, it is likely that clinicians tend to make a tentative diagnosis of dengue in most febrile patients lacking obvious localizing signs to suggest an alternative diagnosis $[29,30]$.

In the diagnosis of DHF, APTT + normal PT $+\mathrm{PC}<100 \times$ $10^{9}$ cells $/ \mathrm{L}, \mathrm{PC}<100 \times 10^{9}$ cells $/ \mathrm{L}+\mathrm{ALT}>40 \mathrm{U} / \mathrm{L}, \mathrm{PC}<100 \times$ $10^{9} \mathrm{cells} / \mathrm{L}+\mathrm{ALT}>40 \mathrm{U} / \mathrm{L}+\mathrm{AST}>40 \mathrm{U} / \mathrm{L}$, prolonged APTT + normal PT + AST $>40 \mathrm{U} / \mathrm{L}$, and prolonged APTT + normal $\mathrm{PT}+\mathrm{ALT}>40 \mathrm{U} / \mathrm{L}$ were found to have a comparable sensitivity and specificity in the diagnosis of DHF. However, when facing a patient with an underlying liver dysfunction due to viral hepatitis and/or fatty liver, APTT + normal PT $+\mathrm{PC}<$ $100 \times 10^{9}$ cells/L is the variable of choice for screening DHF.

Predictive value, a calculation of the percentage of correct negative or correct positive result, is applicable once the prevalence of a disease is taken into consideration [19]. The potential roles played by individual variable/varied combined variables in the diagnoses of the overall dengue/DF/DHF are summarized in Table 4 . While trade-off between sensitivity and specificity, and/or suboptimal PPV/NPV was found at measurements using other variable/varied combined variables, prolonged APTT + normal PT $+\mathrm{PC}<100 \times 10^{9}$ cells/L had a favorable sensitivity, specificity, PPV, and NPV in diagnosis of DF and/or DHF. To make the applicability of these clinical-practice-based laboratory data simplified and user-friendly, we propose prolonged APTT + normal PT + $\mathrm{PC}<100 \times 10^{9}$ cells/L be used for screening and evaluating the likelihood of DF and/or DHF. 
The present study implies that daily-practice-based laboratory data play a complementary role in prompting the suspicion and/or facilitating the diagnosis of DF and/or DHF, which is especially important for clinicians who are inexperienced with these infectious entities. In addition to providing an appropriate therapeutic guidance, a timely suspicion and diagnosis of dengue infection may help the public health authorities launch necessary containment measures earlier, thus diminishing the amplitude of a dengue epidemic that would otherwise be a much larger one.

As DF/DHF features dynamically changing clinical and laboratory manifestations within a few days [14, 16, 31, 32], clinicians may repeatedly sample serum specimens for dailypractice-based laboratory tests if the initial ones do not disclose clear enough information for evaluation of the likelihood of DF/DHF.

Our data were obtained from adult patients during a dengue epidemic due to DENV-2 in Taiwan. Of our serologically dengue-negative patients, $78 \%$ suffered viral infections other than dengue and 6\% suffered bacterial sepsis, while the rest $22 \%$ experienced miscellaneous diseases (see footnote of Table 1 for details). The entities of febrile illness in the nondengue patients might affect the measurements of sensitivity, specificity, PPV, and NPV for dengue illness using the clinical-practice-based laboratory data in our study, and this is one of the limitations that deserves attention. As clinical and laboratory manifestations in $\mathrm{DF} / \mathrm{DHF}$ result from sophisticated immunologic reactions $[17,24,33]$, which may vary from patients in one series to another depending on the genetics of the hosts and the culprit viruses [34, 35], additional limitations of our study must be addressed. It is uncertain whether these daily-practicebased data are applicable in facilitating diagnosis of DF/DHF in adults of other race and/or DF/DHF caused by DENV of other serotypes. Likewise, it is uncertain whether these daily-practice data are applicable in facilitating diagnosis of DF/DHF in pediatric patients. Further study is merited to clarify these important questions, as the answers potentially greatly impact medicine practice in dengue epidemics which are distributed worldwide, mainly in tropical areas where medical resources are deficient.

\section{Ethical Approval}

These data were analyzed anonymously, and the study was conducted with a waiver of patient consent approved by the Institutional Review Board of Chang Gung Memorial Hospital (Document number 99-3533B).

\section{Conflict of Interests}

The authors have declared that they have no conflict of interests.

\section{Acknowledgments}

This work was supported by Grant no. NSC100-2314-B182002-MY3 from the National Science Council, Executive
Yuan, Taiwan. The authors thank Professor Paul L. Chang and Professor S. N. Lu for their helpful comments and the members of the Infection Control Team, Kaohsiung Chang Gung Memorial Hospital, for their assistance in the collection of data.

\section{References}

[1] C.-Y. Tsai, I.-K. Lee, C.-H. Lee, K. D. Yang, and J.-W. Liu, "Comparisons of dengue illness classified based on the 1997 and 2009 World Health Organization dengue classification schemes," Journal of Microbiology, Immunology and Infection, vol. 46, no. 4, pp. 271-281, 2013.

[2] R. V. Gibbons and D. W. Vaughn, "Dengue: an escalating problem," British Medical Journal, vol. 324, no. 7353, pp. 15631566, 2002.

[3] M. G. Guzman and G. Kouri, "Dengue: an update," The Lancet Infectious Diseases, vol. 2, no. 1, pp. 33-42, 2002.

[4] WHO, Dengue Hemorrhagic Fever: Diagnosis, Treatment and Control, World Health Organization, Geneva, Switzerland, 1997.

[5] P.-Y. Shu and J.-H. Huang, "Current advances in dengue diagnosis," Clinical and Diagnostic Laboratory Immunology, vol. 11, no. 4, pp. 642-650, 2004.

[6] M. G. Guzmán, G. Kouri, L. Valdes et al., "Epidemiologic studies on dengue in Santiago de Cuba, 1997," American Journal of Epidemiology, vol. 152, no. 9, pp. 793-799, 2000.

[7] M. P. Mammen Jr., C. Pimgate, C. J. Koenraadt et al., "Spatial and temporal clustering of dengue virus transmission in Thai villages," PLoS Medicine, vol. 5, no. 11, article e205, pp. 16051616, 2008.

[8] J. R. Stephenson, “The problem with dengue," Transactions of the Royal Society of Tropical Medicine and Hygiene, vol. 99, no. 9, pp. 643-646, 2005.

[9] Centers for Disease Control (CDC), CDC Annual Report 2007, Department of Health, Executive Yuan, Taipei, Taiwan, 2007.

[10] J.-W. Liu, B.-S. Khor, C.-H. Lee, I.-K. Lee, R.-F. Chen, and K. D. Yang, "Dengue haemorrhagic fever in Taiwan," Dengue Bulletin, vol. 27, pp. 19-23, 2003.

[11] W.-J. Chen, S.-L. Chen, L.-J. Chien et al., "Silent transmission of the dengue virus in southern Taiwan," American Journal of Tropical Medicine and Hygiene, vol. 55, no. 1, pp. 12-16, 1996.

[12] C.-C. Kan, P.-F. Lee, T.-H. Wen et al., "Two clustering diffusion patterns identified from the 2001-2003 dengue epidemic, Kaohsiung, Taiwan," American Journal of Tropical Medicine and Hygiene, vol. 79, no. 3, pp. 344-352, 2008.

[13] I.-K. Lee, J.-W. Liu, and K. D. Yang, "Clinical characteristics and risk factors for concurrent bacteremia in adults with dengue hemorrhagic fever," American Journal of Tropical Medicine and Hygiene, vol. 72, no. 2, pp. 221-226, 2005.

[14] B.-S. Khor, J.-W. Liu, I.-K. Lee, and K. D. Yang, "Dengue hemorrhagic fever patients with acute abdomen: clinical experience of 14 cases," American Journal of Tropical Medicine and Hygiene, vol. 74, no. 5, pp. 901-904, 2006.

[15] I.-K. Lee, J.-W. Liu, and K. D. Yang, "Clinical and laboratory characteristics and risk factors for fatality in elderly patients with dengue hemorrhagic fever," American Journal of Tropical Medicine and Hygiene, vol. 79, no. 2, pp. 149-153, 2008.

[16] I.-K. Lee, J.-W. Liu, and K. D. Yang, "Clinical characteristics, risk factors, and outcomes in adults experiencing dengue hemorrhagic fever complicated with acute renal failure," American 
Journal of Tropical Medicine and Hygiene, vol. 80, no. 4, pp. 651655, 2009.

[17] H.-Y. Lei, T.-M. Yeh, H.-S. Liu, Y.-S. Lin, S.-H. Chen, and C.-C. Liu, "Immunopathogenesis of dengue virus infection," Journal of Biomedical Science, vol. 8, no. 5, pp. 377-388, 2001.

[18] Y.-H. Wang, A.-S. Lin, Y.-F. Lai, T.-Y. Chao, J.-W. Liu, and S.-F. Ko, "The high value of high-resolution computed tomography in predicting the activity of pulmonary tuberculosis," International Journal of Tuberculosis and Lung Disease, vol. 7, no. 6, pp. 563-568, 2003.

[19] M. H. Zweig and G. Campbell, "Receiver-operating characteristic (ROC) plots: a fundamental evaluation tool in clinical medicine," Clinical Chemistry, vol. 39, no. 4, pp. 561-577, 1993.

[20] M. P. Muller, A. J. Mcgeer, K. Hassan, J. Marshall, and M. Christian, "Evaluation of pneumonia severity and acute physiology scores to predict ICU admission and mortality in patients hospitalized for influenza," PLoS One, vol. 5, no. 3, Article ID e9563, 2010.

[21] R.-F. Chen, J.-W. Liu, W.-T. Yeh et al., "Altered T helper 1 reaction but not increase of virus load in patients with dengue hemorrhagic fever," FEMS Immunology and Medical Microbiology, vol. 44, no. 1, pp. 43-50, 2005.

[22] R.-F. Chen, K. D. Yang, L. Wang, J.-W. Liu, C.-C. Chiu, and J.-T. Cheng, "Different clinical and laboratory manifestations between dengue haemorrhagic fever and dengue fever with bleeding tendency," Transactions of the Royal Society of Tropical Medicine and Hygiene, vol. 101, no. 11, pp. 1106-1113, 2007.

[23] L. Wang, R.-F. Chen, J.-W. Liu, H.-R. Yu, H.-C. Kuo, and K. D. Yang, "Implications of dynamic changes amongst TNF $\alpha$, membrane TNF receptor and soluble TNF receptor levels in regard to the severity of dengue infection," American Journal of Tropical Medicine and Hygiene, vol. 77, no. 2, pp. 297-302, 2007.

[24] A. L. Rothman and F. A. Ennis, "Immunopathogenesis of dengue hemorrhagic fever," Virology, vol. 257, no. 1, pp. 1-6, 1999.

[25] S. Wang, R. He, J. Patarapotikul, B. L. Innis, and R. Anderson, "Antibody-enhanced binding of dengue-2 virus to human platelets," Virology, vol. 213, no. 1, pp. 254-257, 1995.

[26] Y. H. Huang, C. C. Liu, S. T. Wang et al., "Activation of coagulation and fibrinolysis during dengue virus infection," Journal of Medical Virology, vol. 63, no. 3, pp. 247-251, 2001.

[27] S. L. Seneviratne, G. N. Malavige, and H. J. de Silva, "Pathogenesis of liver involvement during dengue viral infections," Transactions of the Royal Society of Tropical Medicine and Hygiene, vol. 100, no. 7, pp. 608-614, 2006.

[28] F. Citarella, A. Felici, M. Brouwer, J. Wagstaff, A. Fantoni, and C. E. Hack, "Interleukin-6 downregulates factor XII production by human hepatoma cell line (HepG2)," Blood, vol. 90, no. 4, pp. 1501-1507, 1997.

[29] D. Chadwick, B. Arch, A. Wilder-Smith, and N. Paton, "Distinguishing dengue fever from other infections on the basis of simple clinical and laboratory features: application of logistic regression analysis," Journal of Clinical Virology, vol. 35, no. 2, pp. 147-153, 2006.

[30] L. Tanner, M. Schreiber, J. G. H. Low et al., "Decision tree algorithms predict the diagnosis and outcome of dengue fever in the early phase of illness," PLoS Neglected Tropical Diseases, vol. 2, no. 3, article e196, 2008.

[31] I.-K. Lee, W.-H. Lee, K. D. Yang, and J.-W. Liu, "Comparison of the effects of oral hydration and intravenous fluid replacement in adult patients with non-shock dengue hemorrhagic fever in Taiwan," Transactions of the Royal Society of Tropical Medicine and Hygiene, vol. 104, no. 8, pp. 541-545, 2010.
[32] I.-K. Lee, J.-W. Liu, and K. D. Yang, "Fatal dengue hemorrhagic fever in adults: emphasizing the evolutionary pre-fatal clinical and laboratory manifestations," PLoS Neglected Tropical Diseases, vol. 6, no. 2, article e1532, 2012.

[33] W. Dejnirattisai, T. Duangchinda, C.-L. S. Lin et al., "A complex interplay among virus, dendritic cells, $\mathrm{T}$ cells, and cytokines in dengue virus infections," Journal of Immunology, vol. 181, no. 9, pp. 5865-5874, 2008.

[34] J. R. Stephenson, "Understanding dengue pathogenesis: implications for vaccine design," Bulletin of the World Health Organization, vol. 83, no. 4, pp. 308-314, 2005.

[35] R.-F. Chen, L. Wang, J.-T. Cheng et al., "Combination of CTLA4 and TGF1 gene polymorphisms associated with dengue hemorrhagic fever and virus load in a dengue- 2 outbreak," Clinical Immunology, vol. 131, no. 3, pp. 404-409, 2009. 

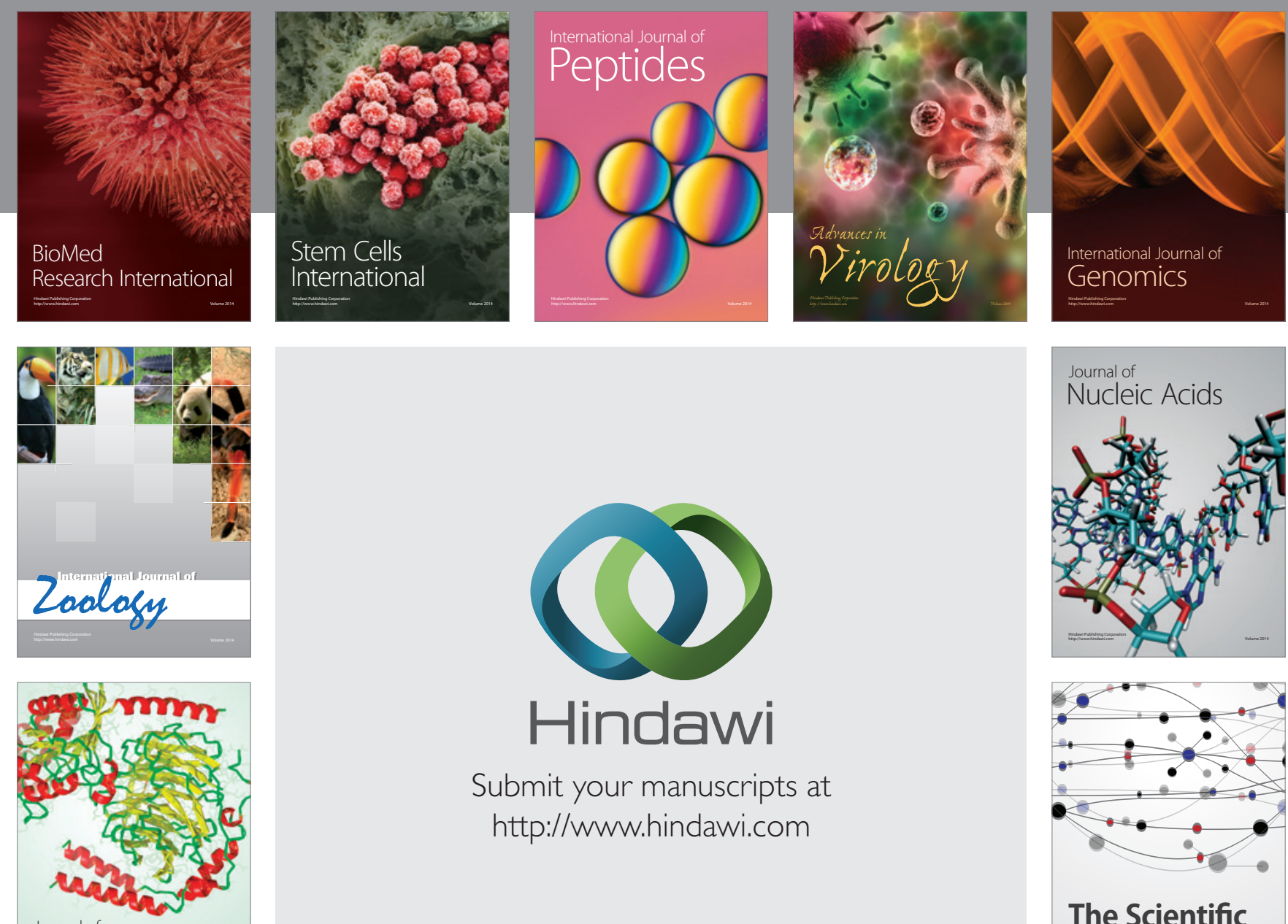

Submit your manuscripts at

http://www.hindawi.com

Journal of
Signal Transduction


The Scientific World Journal
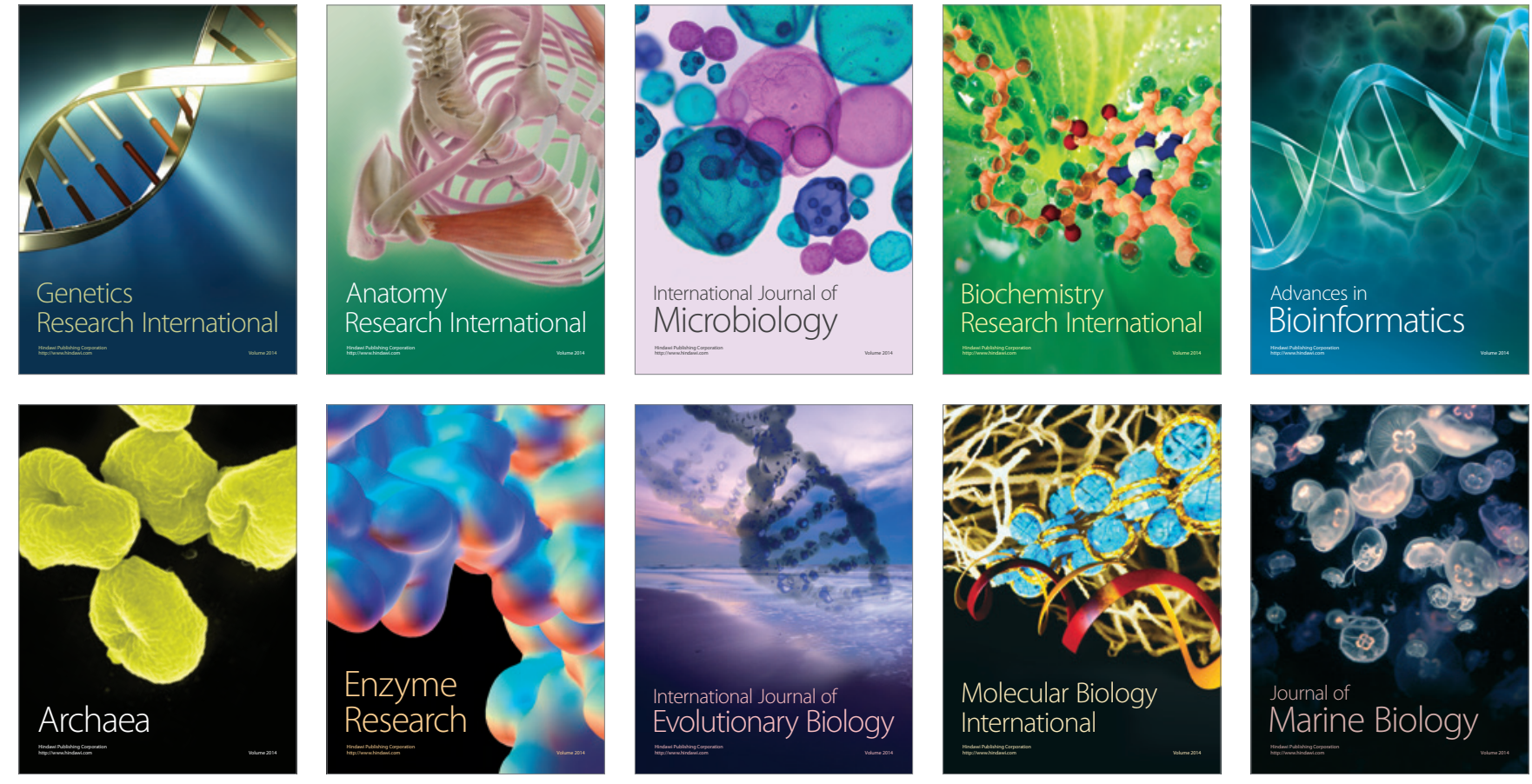